\title{
ON THE APPLICATION OF THE BOREL-CANTELLI LEMMA
}

\author{
BY \\ K. L. CHUNG(1) AND P. ERDÖS
}

Consider a probability space $(\Omega, \mathcal{C}, P)$ and a sequence of events $(\mathcal{C}$-measurable sets in $\Omega)\left\{E_{k}\right\}, k=1,2, \ldots$. The upper (or outer) limiting set of the sequence $\left\{E_{k}\right\}$ is defined by

$$
\lim \sup E_{k}=\bigcap_{n=1}^{\infty} \bigcup_{k=n}^{\infty} E_{k} \text {. }
$$

We recall that the events $E_{k}$ are said to be (mutually) independent (with respect to the probability measure $P$ ) if for any finite number of distinct subscripts $k_{1}, \cdots, k_{s}$ we have

$$
P\left(E_{k_{1}} \cdots E_{k_{s}}\right)=P\left(E_{k_{1}}\right) \cdots P\left(E_{k_{s}}\right) .
$$

The celebrated Borel-Cantelli lemma asserts that

(A) If $\sum P\left(E_{k}\right)<\infty$, then $P\left(\lim \sup E_{k}\right)=0$;

(B) If the events $E_{k}$ are independent and if $\sum P\left(E_{k}\right)=\infty$, then $P\left(\lim \sup E_{k}\right)=1$. In intuitive language $P\left(\lim \sup E_{k}\right)$ is the probability that the events $E_{k}$ occur "infinitely often" and will be denoted by $P\left(E_{k}\right.$ i.o. $)$. This lemma is the basis of all theorems of the strong type in probability theory. Its application is made difficult by the assumption of independence in part (B). As Borel already noticed [1, p. $48 \mathrm{ff}$.], this assumption can be removed if we assume that $\left({ }^{2}\right)$

$$
\sum P\left(E_{k} \mid E_{1}^{\prime} \cdots E_{k-1}^{\prime}\right)=\infty
$$

where $P(F \mid E)$ denotes the conditional probability of $F$ on the hypothesis of $E$ and $E^{\prime}$ denotes the complement of $E$. Although Borel used the condition (0) successfully in his pioneering work on the metric theory of continued fractions, it is too stringent for many purposes. To overcome the difficulty one usually constructs a sequence of independent events out of the given sequence and applies (B) to the new one. This is the device used for instance in the proof of the law of the iterated logarithm and similar theorems. There is however another group of strong theorems to which this method does not

Received by the editors June 14, 1951.

(1) Research supported by an ONR contract.

(2) Clearly we may suppose that $P\left(\bigcup_{j=1}^{k} E_{j}\right)<1$ for every $k$ so that the conditional probability is defined. Added in proof. Conditions like (0) were used a great deal by Paul Lévy and other authors in generalizations to dependent variables; however, that is not what we have in mind here. 
seem to apply. The following theorem furnishes an alternative method which may be of fairly general applicability. On the other hand it does not seem to apply to the law of the iterated logarithm, etc. Two examples given below will serve as illustrations, of which the second concerns the arcsin law.

THEOREM 1. Let $\left\{E_{k}\right\}$ be a sequence of events satisfying:

(i) $\sum P\left(E_{k}\right)=\infty$.

(ii) For every pair of positive integers $h, n$ with $n \geqq h$ there exist $c(h)$ and $H(n, h)>h$ such that for every $k \geqq H(n, h)$ we have

$$
P\left(E_{k} \mid E_{h}^{\prime} \cdots E_{n}^{\prime}\right)>c P\left(E_{k}\right) .
$$

(iii) There exist two absolute constants $c_{1}$ and $c_{2}$ with the following property: to each $E_{j}$ there corresponds a set of events $E_{j_{1}}, \cdots, E_{j_{s}}$ belonging to $\left\{E_{k}\right\}$ such that

$$
\sum_{i=1}^{s} P\left(E_{j} E_{j_{i}}\right)<c_{1} P\left(E_{j}\right)
$$

and if $k>j$ but $E_{k}$ is not among the $E_{j_{i}}(1 \leqq i \leqq s)$ then

$$
P\left(E_{j} E_{k}\right)<c_{2} P\left(E_{j}\right) P\left(E_{k}\right) .
$$

Then $P\left(E_{k}\right.$ i.o. $)=1$.

A defense of the assumptions made seems in order. The conditions (i) and (ii) together resemble Borel's condition ( 0 ) but actually they are very much weaker. The point is that the function $H(n)$ is at our disposal and can be chosen of an infinitely greater order of magnitude then $n$. To put it in a picturesque way, (iii) requires only that the arbitrarily remote past should have no overwhelming effect on the present which is certainly a state of affairs to be hoped for in probability problems. As regards the additional conditions in (iii), they involve only joint probabilities of pairs of events, or what is sometimes referred to as dependence to the second order; part (2) would usually deal with the dependence at close range while (3) deals with the general situation.

Before proceeding to the proof we shall state a simple lemma.

LEMMA. Let $\left\{F_{k}\right\}, k=1, \cdots, N$, be an arbitrary sequence of events in $(\Omega, \mathcal{C}, P)$. We have, if $P\left(\cup_{k=1}^{n} F_{k}\right)>0$,

$$
2 \sum_{1 \leqq i<k \leqq N} P\left(F_{j} F_{k}\right) \geqq\left[P\left(\bigcup_{k=1}^{N} F_{k}\right)\right]^{-1}\left(\sum_{k=1}^{N} P\left(F_{k}\right)\right)^{2}-\sum_{k=1}^{N} P\left(F_{k}\right) .
$$

Proof. Define random variables $X_{k}(\omega), \omega \in \Omega$, as follows:

$$
X_{k}(\omega)=\left\{\begin{array}{lll}
0 & \text { if } & \omega \in F_{k}, \\
1 & \text { if } & \omega \in F_{k} .
\end{array}\right.
$$


The following identity is evident:

(5) $2 \sum_{1 \leq j<k \leqq N} P\left(F_{j} F_{k}\right)=E\left\{\left(X_{1}+\cdots+X_{N}\right)^{2}\right\}-E\left(X_{1}^{2}+\cdots+X_{N}^{2}\right)$.

Now by the Schwarz inequality we have

(6) $\left[E\left(X_{1}+\cdots+X_{N}\right)\right]^{2} \leqq P\left(X_{1}+\cdots+X_{N}>0\right) E\left\{\left(X_{1}+\cdots+X_{N}\right)^{2}\right\}$.

Since $E\left(X_{k}\right)=E\left(X_{k}^{2}\right)=P\left(F_{k}\right), P\left(X_{1}+\cdots+X_{N}>0\right)=P\left(\bigcup_{k=1}^{N} F_{k}\right)$ by definition, (4) follows from (5) and (6).

Proof of Theorem 1. Let

$$
B_{h}=\bigcup_{k=h}^{\infty} E_{k} .
$$

Since $\left(E_{k}\right.$ i.o. $)=\lim _{h \rightarrow \infty} P\left(B_{h}\right)$, it is sufficient to prove that $P\left(B_{h}\right)=1$ for every $h$. Suppose that this is not true for a certain $h$; let $P\left(B_{h}\right)=1-\delta, \delta>0$. Thus

$$
P\left(\bigcap_{k=h}^{\infty} E_{k}^{\prime}\right)=\delta>0 .
$$

Given any $\epsilon, 0<\epsilon<1-\delta$, we can find $n$ such that $P\left(\cup_{k=h}^{n} E_{k}\right)>1-\delta-\epsilon$ so that if we write $D_{h, n}=\bigcup_{k=h}^{\infty} E_{k}-\bigcup_{k=h}^{n} E_{k}$, we have

$$
P\left(D_{h, n}\right)<\epsilon .
$$

We have by (1) and (7), if $k>H(n)$,

$$
P\left(E_{k} E_{h}^{\prime} \cdots E_{n}^{\prime}\right)>c \delta P\left(E_{k}\right) .
$$

Hence by (i), $\sum_{k-H(n)}^{\infty} P\left(E_{k} E_{n}^{\prime} \cdots E_{n}^{\prime}\right)=\infty$. Therefore there exists an integer $H^{\prime}(n)>H(n)$ such that $\left(H=H(n), H^{\prime}=H^{\prime}(n)\right)$

$$
1<\sum_{k=H}^{H^{\prime}} P\left(E_{k} E_{h}^{\prime} \cdots E_{n}^{\prime}\right) \leqq 2 .
$$

From (9) and (10) we obtain

$$
\sum_{k=H}^{H^{\prime}} P\left(E_{k}\right)<\frac{2}{c \delta} .
$$

From (2), (3), and (11) we have

$$
\begin{aligned}
\sum_{H \leqq i<k \leqq H^{\prime}} P\left(E_{j} E_{k}\right) \leqq & c_{1} \sum_{j=H}^{H^{\prime}} P\left(E_{k}\right)+c_{2} \sum_{H \leqq j<k \leqq H^{\prime}} P\left(E_{j}\right) P\left(E_{k}\right) \\
& <\frac{2 c_{i}}{c \delta}+\frac{c_{2}}{2}\left(\frac{2}{c \delta}\right)^{2}=c_{3}(\delta),
\end{aligned}
$$


where $c_{3}(\delta)$ is a constant defined by the last equality.

Now let $F_{k}=E_{k} E_{h}^{\prime} \cdots E_{n}^{\prime}, H \leqq k \leqq H^{\prime}$. It is obvious that $\bigcup_{k=H}^{H^{\prime}} F_{k}$ is a subset of $D_{h, n}$, hence by (8),

$$
P\left(\bigcup_{k=H}^{H^{\prime}} F_{k}\right)<\epsilon .
$$

From (10) and (11) we have

$$
1<\sum_{k=H}^{H^{\prime}} P\left(F_{k}\right) \leqq \sum_{k=H}^{H^{\prime}} P\left(E_{k}\right)<\frac{2}{c \delta} .
$$

Applying the lemma to $\left\{F_{k}\right\}, H \leqq k \leqq H^{\prime}$, we obtain using (13) and (14)

$$
2 \sum_{H \leqq j<k \leqq H^{\prime}} P\left(E_{j} E_{k}\right) \geqq 2 \sum_{H \leqq j<k \leqq H^{\prime}} P\left(F_{j} F_{k}\right) \geqq \frac{1}{\epsilon}-\frac{2}{c \delta} .
$$

But (12) and (15) are incompatible for sufficiently small $\epsilon$. This contradiction proves that $\delta=0$. Hence $P\left(B_{h}\right)=1$. q.e.d.

In the two applications given below we shall treat only the simplest Bernoullian case, since we are more interested in the principle involved than the technical difficulties. It is not hard to generalize Theorems 2 and 3 to fairly general lattice cases or even continuous cases. It will be seen from their proofs that only certain asymptotic formulas and a kind of boundedness of $S_{n}$, with probability one or even in probability, are required. These are available in more general cases, thanks to various modern limit theorems.

Theorem 2. Let $\left\{X_{k}\right\}, k=1,2, \cdots$, be independent random variables and each $X_{k}$ assume the values +1 and -1 with probabilities $1 / 2$ and $1 / 2$. Let $S_{n}=\sum_{k=1}^{n} X_{k}$. Let $\left\{n_{i}\right\}, i=1,2, \cdots$, be an increasing sequence of even integers such that there exists an absolute constant $A$ with the property that

$$
n_{i+1}-n_{i}>A n_{i}^{1 / 2} \text {. }
$$

Then

$$
P\left(S_{n_{i}}=0 \text { i.o. }\right)=\left\{\begin{array}{l}
0 \\
1
\end{array}\right.
$$

according as

$$
\sum_{i} n_{i}^{-1 / 2}\left\{\begin{array}{l}
< \\
=
\end{array}\right\} \infty
$$

REMARK. The theorem in the divergence case is not true without some such condition as (16). Example: Take $\left\{n_{i}\right\}$ to be the sequence of even integers in the intervals $\left[k^{8}, k^{8}+k^{5}\right], k=1,2, \cdots$. For an alternative condi- 
tion and proof see [3, p. 1009].

Proof. The convergence case follows directly from part (A) of the BorelCantelli lemma without the condition (16).

Next, let $E_{i}$ denote the event $S_{n_{i}}=0$. We know that

$$
P\left(E_{i}\right)=P\left(S_{n_{i}}=0\right) \sim\left(2 / \pi n_{i}\right)^{1 / 2} .
$$

Hence condition (i) in Theorem 1 is satisfied.

To verify the condition (ii) in Theorem 1 we notice that $\left|S_{n_{i}}\right| \leqq n_{i}$, hence

$$
\begin{aligned}
P\left(S_{n_{k}}=0 \mid S_{n_{h}}\right. & \left.\neq 0, \cdots, S_{n_{i}} \neq 0\right) \\
& \geqq \operatorname{Min}_{|x| \leqq n_{i}} P\left(S_{n_{k}}=0 \mid S_{n_{i}}=x\right)=\operatorname{Min}_{|x| \leqq n_{i}} P\left(S_{n_{k}}-S_{n_{i}}=-x\right) \\
& =\operatorname{Min}_{|x| \leqq n_{i}} P\left(S_{n_{k}-n_{i}}=-x\right) .
\end{aligned}
$$

Now we have, if $x^{2}=o(n)$,

$$
P\left(S_{n}=x\right)=C\left(\begin{array}{c}
n \\
(n-x) / 2
\end{array}\right) \frac{1}{2^{n}} \sim\left(\frac{2}{\pi n}\right)^{1 / 2} e^{-x^{2} / 2 n} \sim\left(\frac{2}{\pi n}\right)^{1 / 2} .
$$

We choose $H(i)$ sufficiently large so that if $k>H(i)$, then $n_{i}^{2}=o\left(n_{k}\right)$. Then we have for all $|x| \leqq n_{i}$,

$$
P\left(S_{n_{k}-n_{i}}=-x\right) \sim\left(\frac{2}{\pi n_{k}}\right)^{1 / 2} .
$$

Therefore we have for all $h, i \geqq h$ and $k \geqq H(i)$ and any fixed $c<1$, if $H(1)$ is sufficiently large,

$$
P\left(S_{n_{k}}=0 \mid S_{n_{h}} \neq 0, \cdots, S_{n_{i}} \neq 0\right)>c P\left(S_{n_{k}}=0\right) .
$$

Thus condition (ii) in Theorem 1 is satisfied.

To verify the condition (iii) in Theorem 1 we have

$$
\begin{aligned}
P\left(E_{j} E_{k}\right) & =P\left(S_{n_{j}}=0\right) P\left(S_{n_{k}-n_{j}}=0\right) \\
& \sim P\left(S_{n_{j}}=0\right)\left(\frac{2}{\pi\left(n_{k}-n_{j}\right)}\right)^{1 / 2} \\
& \sim P\left(S_{n_{j}}=0\right) P\left(S_{n_{k}}=0\right)\left(\frac{n_{k}}{n_{k}-n_{j}}\right)^{1 / 2} \\
& =P\left(E_{j}\right) P\left(E_{k}\right)\left(\frac{n_{k}}{n_{k}-n_{j}}\right)^{1 / 2} .
\end{aligned}
$$

If $n_{k}>2 n_{j}$, then

$$
\left(\frac{n_{k}}{n_{k}-n_{j}}\right)^{1 / 2}<2^{1 / 2}
$$


We call the events $E_{k}$ with $n_{j}<n_{k} \leqq 2 n_{j}$ the events $E_{j_{i}}(1 \leqq i \leqq s)$ associated with each $E_{j}$. We have as before

$$
\sum^{\prime} P\left(E_{j} E_{k}\right) \sim P\left(E_{j}\right) \sum^{\prime}\left(n_{k}-n_{j}\right)^{-1 / 2}
$$

where the summation extends to those $k$ for which $n_{j}<n_{k} \leqq 2 n_{j}$. From (16) we deduce that if $k>j$ ( $A_{1}$ denoting an absolute constant),

$$
n_{k}-n_{j}>A_{1}\left(k^{2}-j^{2}\right) \text {. }
$$

Let $N$ denote the number of $k$ 's satisfying $n_{j}<n_{k} \leqq 2 n_{j}$. From the last inequality we deduce that $n_{j}+A_{1}\left(N^{2}+2 j N\right) \leqq n_{j+N} \leqq 2 n_{j}$. Hence we have

$$
N \leqq\left(\frac{n_{j}}{A_{1}}\right)^{1 / 2} \text {. }
$$

Now using the Schwarz inequality, (16), and (20) we obtain

$$
\left(\sum^{\prime}\left(n_{k}-n_{j}\right)^{-1 / 2}\right)^{2} \leqq N \sum^{\prime}\left(n_{k}-n_{j}\right)^{-1} \leqq\left(\frac{n_{j}}{A_{1}}\right)^{1 / 2} \frac{1}{A n_{j}^{1 / 2}}=\left(\frac{1}{A_{1} A^{2}}\right)^{1 / 2}
$$

(19) and (21) give (2) while (17) and (18) give (3). q.e.d.

TheOREM 3. Let $\left\{X_{n}\right\}$ be as in Theorem 2 and let $N_{n}$ denote the number of positive terms among $S_{1}, \cdots, S_{n}$. Let $\phi(n)$ be an increasing function of $n$. Then

$$
P\left(N_{n} \leqq \frac{n}{\phi(n)} \text { i.o. }\right)=\left\{\begin{array}{l}
0 \\
1
\end{array}\right.
$$

according as

$$
\sum \frac{1}{n(\phi(n))^{1 / 2}}\left\{\begin{array}{l}
< \\
=
\end{array}\right\} \infty .
$$

REMARK. This is the strong theorem corresponding to the now celebrated arcsin law. On grounds of symmetry we may replace the left side in (22) by $P\left(N_{n} \geqq n(1-1 / \phi(n))\right.$ i.o. $)$.

Proof. Standard arguments $\left(^{3}\right)$ show that we may suppose that $\phi(n) \leqq n^{\text {e }}$ for some $0<\epsilon<1 / 2$. The convergence case follows easily from the arcsin law for Bernoullian variables (see [2, p. 252]; the convention made there regarding the "positiveness" of $S_{n}$ makes no difference in the asymptotic formula below), which asserts that

$$
P\left(N_{n} \leqq \frac{n}{\phi(n)}\right) \sim \frac{2}{\pi(\phi(n))^{1 / 2}} .
$$

To prove the theorem in the divergence case we note first that the di(3) Cf e.g. [3. p. 1010]. 
vergence of the series in (23) implies that of

$$
\sum_{n} \frac{1}{n\left(\phi\left(n^{r}\right)\right)^{1 / 2}}
$$

for any $r>0$ (proof by the integral test). Let $\phi\left(n^{2}\right)=\psi(n)$. Define $E_{k}$ to be the event

$$
S_{2 k}=0, \quad S_{i}<0 \quad \text { for } 2 k<i \leqq 2 k \psi(k) .
$$

Obviously $E_{k}$ implies that $N_{2 k \psi(k)} \leqq 2 k$. Writing $2 k \psi(k)=n$, we have, since $\psi(k) \leqq k^{2 \epsilon}, k \geqq n^{\prime}$ where $n^{\prime}=\left(2^{-1} n\right)^{1 /(1+2 \epsilon)}$. Hence $N_{n} \leqq n / \psi\left(n^{\prime}\right)$. For all sufficiently large $n, \psi\left(n^{\prime}\right) \geqq \phi(n)$. Hence in order to prove the second part in (22) it is sufficient to prove that $P\left(E_{k}\right.$ i.o. $)=1$.

It is known that (see e.g. $\left[2\right.$, p. 252]) $P\left(S_{i}<0\right.$ for $\left.0<i \leqq n\right) \sim b n^{-1 / 2}$ for some absolute constant $b>0$. Hence we have

$$
\begin{aligned}
P\left(E_{k}\right) & =P\left(S_{2 k}=0\right) P\left(S_{i}<0 \text { for } 0<i \leqq 2 k \psi(k)-2 k\right) \\
& \sim b k^{-1 / 2}(k \psi(k))^{-1 / 2}=b k^{-1}(\psi(k))^{-1 / 2} .
\end{aligned}
$$

Hence condition (i) in Theorem 1 is satisfied.

To verify condition (ii) in Theorem 1 we note that (without loss of generality we may suppose $n \psi(n)$ to be an integer for all $n$ ), if $k>H(n)$,

$$
\begin{aligned}
P\left(E_{k} \mid S_{1}\right. & \left.=x_{1}, \cdots, S_{2 n \psi(n)}=y\right) \\
& =\sum_{x} P\left(E_{k} \mid S_{H(n)}=x\right) P\left(S_{H(n)}=x \mid S_{2 n \psi(n)}=y\right) \\
& =\sum_{x} P\left(E_{k} \mid S_{H(n)}=x\right) P\left(S_{H(n)-2 n \psi(n)}=x-y\right)
\end{aligned}
$$

where $x_{1}, \cdots, y, x$ are integers. Now $|y| \leqq 2 n \psi(n)$, hence if we choose $H(n)$ sufficiently large, $P\left(S_{H(n)-2 n \psi(n)}=x-y\right) \sim P\left(S_{H(n)}=x\right)$ as $n \rightarrow \infty$, at least if $x$ is within a certain range, say $|x| \leqq H(n)^{1 / 2+\eta}, \eta>0$. (This is because of the limitations of the Gaussian approximation.) But the other range of $x$ is negligible in the sense that

$$
\begin{aligned}
\sum_{|x|>H(n)^{1 / 2+\eta}} P\left(S_{H(n)-2 n \psi(n)}=x-y\right) & \\
= & O\left(\sum_{|x| \leqq H(n)^{1 / 2+\eta}} P\left(S_{H(n)-2 n \psi(n)}=x-y\right)\right) .
\end{aligned}
$$

Hence we have

$$
\begin{aligned}
\operatorname{Min}_{|y| \leqq 2 n \psi(n)} P\left(E_{k} \mid S_{1}=x_{1}, \cdots,\right. & \left.S_{2 n \psi(n)}=y\right) \\
& \sim \sum_{x} P\left(E_{k} \mid S_{H(n)}=x\right) P\left(S_{H(n)}=x\right)=P\left(E_{k}\right) .
\end{aligned}
$$


This implies condition (ii) in Theorem 1.

To verify condition (iii) in Theorem 1 , let $j<k$. If $k \leqq j \psi(j)$, then $P\left(E_{j} E_{k}\right)$ $=0$. If $k>j \psi(j)$ we have

$$
\begin{aligned}
& P\left(E_{k} \mid E_{j}\right)=P\left(S_{2 k}=0 \mid S_{2 j}=0, S_{i}<0 \text { for } 2 j<i \leqq 2 j \psi(j)\right) . \\
& P\left(S_{i}<0 \text { for } 2 k<i \leqq 2 k \psi(k) \mid S_{2 k}=0\right)=P_{1} \cdot P_{2} .
\end{aligned}
$$

Now for every $x$ we have

$$
P\left(S_{2 k}=0 \mid S_{2 j \psi(j)}=x\right)=P\left(S_{2 k-2 j \psi(j)}=-x\right) \leqq b(k-j \psi(j))^{-1 / 2} .
$$

$P_{1}$ being a probability mean of such probabilities we have

$$
P_{1} \leqq b(k-j \psi(j))^{-1 / 2} \text {. }
$$

As for $P_{2}$ we have as in (24),

$$
P_{2} \sim b(2 k \psi(k)-2 k)^{-1 / 2} \sim b(2 k \psi(k))^{-1 / 2} .
$$

Therefore we obtain from (25),

$$
\left.P\left(E_{j} E_{k}\right) \leqq b_{1} P\left(E_{j}\right)\left(k-j \psi^{\prime} j\right)\right)^{-1 / 2}(k \psi(k))^{-1 / 2},
$$

where $b_{1}$ (as $b_{2}, b_{3}$ later) is an absolute constant. Now for every $E_{j}$ we define $E_{j_{i}}, 1 \leqq i \leqq s$, to be those $E_{k}$ with $j \psi(j)<k \leqq 2 j \psi(j)$. We have then by (26)

$$
\begin{aligned}
\sum_{i=1}^{8} P\left(E_{j} E_{j_{i}}\right) & \leqq b_{1} P\left(E_{j}\right)(k \psi(k))^{-1 / 2} \sum_{i=1}^{j \psi(j)} i^{-1 / 2} \\
& \leqq b_{2} P\left(E_{j}\right) .
\end{aligned}
$$

On the other hand if $k>2 j \psi(j)$, then $k-j \psi(j)>k / 2$, hence by (26) and (24)

$$
P\left(E_{j} E_{k}\right) \leqq b_{3} P\left(E_{j}\right) P\left(E_{k}\right) \text {. }
$$

Therefore condition (iii) in Theorem 1 is satisfied. q.e.d.

\section{REFERENCES}

1. E. Borel, Traité du calcul des probabilités et de ses applications, vol. 2, no. 1, Applications à l'arithmétique et da la theorie des fonctions, Paris, Gauthier-Villars, 1926.

2. W. Feller, An introduction to probability theory and its applications, vol. 1, New York, Wiley, 1950.

3. K. L. Chung and P. Erdös, On the lower limit of sums of independent random variables, Ann. of Math. vol. 48 (1947) p. 1003.

Columbia University,

NEW YORK, N. Y.

University of Aberdeen,

Aberdeen, Scotland. 